\title{
Frobenius Manifolds near the Discriminant and Relations in the Tautological Ring
}

\author{
Felix Janda
}

April 9, 2020

\begin{abstract}
We give a criterion for extending a generically semisimple (not necessarily conformal) Frobenius manifold locally near a smooth point of the discriminant to a cohomological field theory.

As an application, we show that a large set of tautological relations related to the Givental-Teleman classification for any generically semisimple cohomological field theories follow from Pixton's generalized Faber-Zagier relations.
\end{abstract}

\section{Introduction}

\subsection{Frobenius manifolds}

Introduced by Dubrovin in 4, Frobenius manifolds are particular flat Riemannian manifolds with an algebra structure on the space of vector fields. The most important examples come from genus zero Gromov-Witten theory where the algebra structure is given by quantum multiplication, and singularity theory where instead the Milnor ring with its multiplicative structure is considered.

A point $p$ in a Frobenius manifold $M$ is called semisimple if there exists a basis of idempotent local vector fields $\epsilon_{i}$ near $p$ which are orthogonal with respect to the metric $\eta$, and can be integrated to give the so-called canonical coordinates. The local behavior near a semisimple point is uniquely determined by the norms $\eta\left(\epsilon_{i}, \epsilon_{i}\right)$ of the idempotents with respect to the metric. Dubrovin has shown that the $\eta\left(\epsilon_{i}, \epsilon_{i}\right)$ satisfy the system of Darboux-Egoroff equations and conversely constructs from any generic solutions to the Darboux-Egoroff system a Frobenius manifold.

It is a natural question to ask how this local classification extends to nonsemisimple points. A first step in this direction has been taken by Hertling [10] who shows that the germ of an $N$-dimensional generically semisimple Frobenius

\footnotetext{
Keywords. Frobenius manifolds, Discriminant, Cohomological field theories, Tautological ring
}

2010 Mathematics Subject Classification. 53D45, 14H10 
manifold (or more generally a massive $F$-manifold) near a smooth point $p$ on the discriminant locus of a generically semisimple Frobenius manifold is of the form $I_{2}(m) \times A_{1}^{N-2}$ for some integer parameter $m \geq 3$. This means in particular that all but two idempotent vector fields extend to $p$, and the parameter $m$ describes the behavior of the remaining two idempotents behave near $p$. In this article, Frobenius manifolds such that $m=3$ will be of most importance.

\subsection{Cohomological field theories}

Cohomological fields theories (CohFTs) as first introduced in [16 can be viewed as higher genus analogs of Frobenius manifolds. A CohFT $\Omega$ defined on a vector space $V$ is a system of multilinear maps

$$
\Omega_{g, n}:\left(V^{*}\right)^{\otimes n} \rightarrow H^{*}\left(\bar{M}_{g, n}\right)
$$

to the cohomology of the moduli space of curves $H^{*}\left(\bar{M}_{g, n}\right)$ satisfying some compatibility axioms. By restricting to genus zero, the structure of a formal neighborhood of a point in a Frobenius manifold with tangent space $V$ can be extracted. Under convergence assumptions, $V$ is the tangent space of a point of an actual Frobenius manifold and using a shifting construction $\Omega_{g, n}$ can be extended to a family of CohFTs depending on a point on a Frobenius manifold. We call such a family of CohFTs a convergent CohFT.

For a convergent CohFT $\Omega_{g, n}$, the Givental-Teleman reconstruction [24] of semisimple CohFTs gives a way to reconstruct the CohFT $\Omega_{g, n}$ from its underlying Frobenius manifold $M$ near a semisimple point of $M$ up to a choice of integration constants. In many applications, $\Omega_{g, n}$ is in addition homogeneous with respect to an Euler vector field, in which case, there is a unique choice for the integration constants.

The Givental-Teleman reconstruction gives a way to extend the neighborhood of a semisimple point of a Frobenius manifold to a convergent CohFT. Using Hertling's result, we show in Theorem 3.4.1 that it is also possible to extend the neighborhood of a smooth point on the discriminant locus of an $N$-dimensional Frobenius manifold $M$ to a convergent CohFT, provided that $m=3$. Roughly, the CohFT is constructed by deforming the CohFT corresponding to the $A_{2} \times\left(A_{1}\right)^{N-2}$-singularity using the action of an $R$-matrix constructed from $M$. We should note that special cases of Theorem 3.4.1 on the level of intersection numbers have previously appeared in the literature, such as [1] (extension to genus one), 18, (extension for CohFTs from singularity theory), 3] (extension for CohFTs from Gromov-Witten theory) 1 Compared to the existing literature, Theorem 3.4.1 works on the cycle level and does not depend on the existence of an Euler vector field.

\footnotetext{
${ }^{1}$ It was also communicated to the author that Dubrovin-Liu-Zhang have a proof for the general conformal case on the level of intersection numbers.
} 


\subsection{Tautological relations}

The initial motivation to this work is the study of relations in the tautological ring of the moduli space of curves.

The tautological rings $R H^{*}\left(\bar{M}_{g, n}\right)$ are certain subrings of the cohomology rings $H^{*}\left(\bar{M}_{g, n}\right)$ of the moduli space $\bar{M}_{g, n}$ of stable curves. Starting from the 1980s with Mumford's seminal article [19], they have been studied extensively. However, their structure is still not completely understood: While there is an explicit set of generators parameterized by decorated graphs, the set of relations between the generators is not known. On the other hand, Pixton's set 23] of generalized Faber-Zagier relations gives a well-tested conjectural description for this set of relations. Another conjectural description had been given by Faber's Gorenstein conjecture but it is now known to be false in general [22].

In 20] the relations of Pixton have been shown to arise in the computation of Witten's 3-spin class, or equivalently the CohFT corresponding to the $A_{2}$-singularity, via the Givental-Teleman reconstruction of semisimple CohFTs. The formula that Pandharipande-Pixton-Zvonkine obtain for Witten's 3-spin class has the form of a limit $\phi \rightarrow 0$ of a Laurent series in a variable $\phi$ whose coefficients are tautological classes. The existence of the limit implies cancellation between tautological classes such that no poles in $\phi$ are left in the end. These relations between tautological classes, after adding relations directly following from them, give exactly the relations of Pixton.

As noted in 20, the limit $\phi \rightarrow 0$ can be viewed as approaching a nonsemisimple point on the Frobenius manifold corresponding to the $A_{2}$-singularity. In particular, the same procedure can be applied to get relations from other generically semisimple, convergent CohFTs but it is not clear how the relations from different CohFTs relate to each other. In 14 first comparison results have been proven: The relations from the equivariant Gromov-Witten theory of $\mathbb{P}^{1}$ are equivalent to the relations from the $A_{2}$-theory and in general the relations from equivariant $\mathbb{P}^{N-1}$ imply the $A_{N}$-relations.

While the tautological relations obtained from different CohFTs look very different and are usually more complicated than Pixton's relations, as an application of Theorem 3.4.1 we show in Theorem 3.3.6 that they give the same set of relations. This says in some sense that the relations of Pixton are the universal relations necessary in order for the Givental-Teleman classification to admit non-semisimple limits. Theorem 3.3.6 can also be used to relate more geometric relations to Pixton's relations (see for example 2]).

In this paper we work over $\mathbb{C}$ and with the tautological ring in cohomology. It is actually more natural to define the tautological ring in Chow and the results of this paper apply also to Chow provided that the Givental-Teleman reconstruction is proven in Chow for the relevant (Chow-valued) CohFTs.

\section{Plan of the paper}

In Section 2 we first recall basic properties of Frobenius manifolds, and then state Hertling's classification in Theorem 2.2.2. In Section 3, we start by recalling 
the definition of cohomological field theories and the statement of the GiventalTeleman classification. After that, in Section 3.3, we discuss the tautological ring and the relations resulting from the classification. In Section 3.4 we prove Theorem 3.4.1 about the local extension of semisimple Frobenius manifolds. We discuss in Section 3.5 how its proof implies Theorem 3.3.6 on the comparison of tautological relations. In Section [3.6 we shortly consider the problem of finding a global extension theorem similar to Theorem 3.4.1. Afterwards, in Section 3.7 we study two examples, which illustrate obstructions to directly generalizing our results. In the final Section 3.8 we show that certain other relations obtained from the equivariant Gromov-Witten theory of toric targets can also be expressed in terms of Pixton's relations.

\section{Acknowledgments}

The author is very grateful for various discussions with A. Buryak, E. Clader, Y.P. Lee, D. Petersen, R. Pandharipande, C. Schiessl, S. Shadrin and D. Zvonkine.

The author has learned about the method of obtaining relations by studying a CohFT near the discriminant from D. Zvonkine at the conference Cohomology of the moduli space of curves organized by the Forschungs-institut für Mathematik at ETH Zürich in 2013. D. Zvonkine there also expressed an idea why we should obtain the same relations from different CohFTs. Together with S. Shadrin he studied them in comparison to relations obtained from degree considerations (see Remark 3.3.8).

The results on the extension of a Frobenius manifold to a CohFT is motivated from discussions with M. Kazarian, T. Milanov and D. Zvonkine at the workshop Geometric Invariants and Spectral Curves at the Lorentz Center in Leiden.

Special thanks are due to an anonymous referee for drawing the author's attention to Hertling's work on Frobenius manifolds.

This research was carried out while being a PhD. student of R. Pandharipande at ETH Zürich and being supported by the Swiss National Science Foundation grant SNF 200021_143274.

\section{Frobenius manifolds}

\subsection{Definition and basic properties}

Frobenius manifolds have been introduced by Dubrovin 44. They naturally arise when studying genus zero Gromov-Witten theory. Let us begin by recalling their basic properties in the following slightly redundant definition.

Definition 2.1.1. An $N$-dimensional (complex, even) Frobenius manifold is a 4-tuple $(M, \eta, A, \mathbf{1})$, consisting of

- $M$, a complex, connected manifold of dimension $N$,

- a nonsingular metric $\eta \in \Gamma\left(\operatorname{Sym}^{2}\left(T^{*} M\right)\right)$, 
- a tensor $A \in \Gamma\left(\operatorname{Sym}^{3}\left(T^{*} M\right)\right)$,

- a vector field $\mathbf{1} \in \Gamma(T M)$,

satisfying the following properties:

- A commutative, associative product $\star$ on $T M$, with unit $\mathbf{1}$, is defined by setting for local vector fields $X$ and $Y$ that

$$
\eta(X \star Y, Z)=A(X, Y, Z)
$$

for any local vector field $Z$.

- The metric $\eta$ is flat and $\mathbf{1}$ is an $\eta$-flat vector field.

- Locally around each point there exist flat coordinates $t_{\alpha}$ such that the metric and the unit vector field are constant when written in the basis of the corresponding local vector fields $\frac{\partial}{\partial t_{\alpha}}$.

- Locally on $M$ there exists a holomorphic function $\Phi$ called potential such that

$$
A\left(\frac{\partial}{\partial t_{\alpha}}, \frac{\partial}{\partial t_{\beta}}, \frac{\partial}{\partial t_{\gamma}}\right)=\frac{\partial^{3} \Phi}{\partial t_{\alpha} \partial t_{\beta} \partial t_{\gamma}} .
$$

Definition 2.1.2. A Frobenius manifold is called conformal if it admits an Euler vector field, i.e. a vector field $E$ of the form

$$
E=\sum_{\mu}\left(\alpha_{\mu} t_{\mu}+\beta_{\mu}\right) \frac{\partial}{\partial t_{\mu}}
$$

such that the quantum product, the unit and the metric are eigenfunctions of the Lie derivative $L_{E}$ with eigenvalues $1,-1$ and $2-\delta$ respectively. Here $\delta$ is a rational number called conformal dimension.

\subsection{Discriminant and semisimplicity}

We say that a Frobenius manifold $M$ is (generically) semisimple if the algebra $\left(T_{p} M, \star_{p}\right)$ is semisimple for a generic point $p$ on $M$. The set of points $p \in M$ such that $\left(T_{p} M, \star_{p}\right)$ is not semisimple is called the discriminant locus (or the caustic).

If $M$ is semisimple, near any semisimple point, we can choose a basis $\frac{\partial}{\partial u_{i}}$ of orthogonal idempotents and we use the notation $\Delta_{i}^{-1}$ for their norms. Then

$\Delta_{i}^{\frac{1}{2}} \frac{\partial}{\partial u_{i}}$ define normalized idempotents. As the notation suggests, the vector fields $\frac{\partial}{\partial u_{i}}$ commute and we can integrate them locally near semisimple points to give the canonical coordinates $u_{i}$.

For this article, the structure of a Frobenius manifold near a smooth point of the discriminant plays an important role. We discuss a two-dimensional motivational example before recalling an important result in this direction due to C. Hertling. 
Example 2.2.1. The Givental-Saito theory of the $A_{2}$-singularity, which appears in the study of Witten's 3-spin class, concerns a two-dimensional Frobenius manifold. As a manifold, it is isomorphic to $\mathbb{C}^{2}$ with coordinates $t_{0}, t_{1}$ and its points correspond to versal transformations

$$
\frac{x^{3}}{3}-t_{1} x+t_{0}
$$

of the $A_{2}$-singularity $\frac{x^{3}}{3}$. In the basis $\frac{\partial}{\partial t_{0}}, \frac{\partial}{\partial t_{1}}$ the metric $\eta$ is given by the matrix

$$
\left(\begin{array}{ll}
0 & 1 \\
1 & 0
\end{array}\right)
$$

and the potential is

$$
\Phi\left(t_{0}, t_{1}\right)=\frac{1}{2} t_{0}^{2} t_{1}+\frac{1}{24} t_{1}^{4}
$$

Therefore $\frac{\partial}{\partial t_{0}}$ is the unit, and the only interesting quantum product is

$$
\frac{\partial}{\partial t_{1}} \star \frac{\partial}{\partial t_{1}}=t_{1} \frac{\partial}{\partial t_{0}}
$$

Hence on a two-fold cover of $\mathbb{C}^{2}$ ramified along the discriminant locus $\left\{t_{1}=0\right\}$ we can define the meromorphic idempotents

$$
\epsilon_{ \pm}= \pm \frac{1}{2 \sqrt{t_{1}}} \frac{\partial}{\partial t_{1}}+\frac{1}{2} \frac{\partial}{\partial t_{0}} .
$$

A choice of corresponding canonical coordinates is given by

$$
u_{ \pm}=t_{0} \pm \frac{2}{3} t_{1}^{\frac{3}{2}}
$$

Notice that we can recover the flat vector fields by setting

$$
\frac{\partial}{\partial t_{0}}=\epsilon_{+}+\epsilon_{-}
$$

and

$$
\frac{\partial}{\partial t_{1}}=\left(\frac{3}{4}\left(u_{+}-u_{-}\right)\right)^{\frac{1}{3}}\left(\epsilon_{+}-\epsilon_{-}\right) .
$$

Theorem 2.2.2. ([10, 11]) Let $M$ be a (generically) semisimple Frobenius manifold. In a neighborhood $U$ of a smooth point of the discriminant of $M$, there exists an integer $m \geq 3$ such that

- all but two idempotents $\frac{\partial}{\partial u_{1}}, \frac{\partial}{\partial u_{2}}$ extend holomorphically to the discriminant in $U$,

- for a suitable choice of integration constants, there is a holomorphic root $\left(u_{1}-u_{2}\right)^{2 / m}$, and its vanishing locus describes the discriminant in $U$, 
- The vector fields

$$
\left(u_{1}-u_{2}\right)^{(m-2) / m}\left(\frac{\partial}{\partial u_{1}}-\frac{\partial}{\partial u_{2}}\right), \frac{\partial}{\partial u_{1}}+\frac{\partial}{\partial u_{2}}, \frac{\partial}{\partial u_{\geq 3}}
$$

extend holomorphically to the discriminant and span the tangent space at every point of $U$. The first of these vector fields spans the space of nilpotent tangent vectors at $p$.

Furthermore, if in addition for any flat vector field $X$, for a genus one potential $G$, the function $\mathrm{d} G(X)$ extends to the discriminant, the integer $m$ has to be equal to 3 .

Here, we recall the formula (see [6]) for a genus one potential $G$

$$
\mathrm{d} G=\frac{1}{48} \sum_{i} \mathrm{~d} \log \left(\Delta_{i}\right)+\frac{1}{2} \sum_{i} r_{i i} \mathrm{~d} u_{i}
$$

in which the functions $r_{i}$, , determined up to an integration constant, satisfy

$$
\mathrm{d} r_{i i}=\frac{1}{4} \sum_{j} \frac{\partial \log \left(\Delta_{j}\right)}{\partial u_{i}} \frac{\partial \log \left(\Delta_{i}\right)}{\partial u_{j}}\left(\mathrm{~d} u_{j}-\mathrm{d} u_{i}\right) .
$$

Proof of Theorem 2.2.2. Semisimple Frobenius manifolds are examples of massive $F$-manifolds. By Theorem [10, Theorem 12.2] the germs of a massive $F$ manifolds near a smooth point of the discriminant locus is of type $I_{2}(m) \times A_{1}^{n-2}$. This implies the first part of the Theorem.

Theorem [11, Theorem 6.3] considers the $G$-function of semisimple Frobenius manifolds of type $I_{2}(m)$, and shows that $m$ must be equal to 3 when the $G$ function extends to the discriminant locus 3

\section{Cohomological Field Theories}

\subsection{Definitions}

Cohomological field theories were first introduced by Kontsevich and Manin in [16] (see also 20]) to formalize the structure of classes from Gromov-Witten theory. Let $V$ be an $N$-dimensional $\mathbb{C}$-vector space and $\eta$ a nonsingular bilinear form on $V$.

Before stating their definition, we recall the moduli space $\bar{M}_{g, n}$ of stable, connected, at most nodal algebraic curves of arithmetic genus $g$ with $n$ markings, as well as that there are tautological maps induced by forgetting a marking and gluing along markings:

$$
\begin{aligned}
\bar{M}_{g, n+1} & \rightarrow \bar{M}_{g, n}, \\
\bar{M}_{g_{1}, n_{1}+1} \times \bar{M}_{g_{2}, n_{2}+1} & \rightarrow \bar{M}_{g_{1}+g_{2}, n_{1}+n_{2}}, \\
\bar{M}_{g, n+2} & \rightarrow \bar{M}_{g+1, n}
\end{aligned}
$$

\footnotetext{
${ }^{2}$ They correspond to the diagonal entries of the linear part of the $R$-matrix.

${ }^{3}$ The proof of [11] Theorem 6.3] does not depend on the existence of an Euler vector field.
} 
Definition 3.1.1. A cohomological field theory (CohFT) $\Omega$ on $(V, \eta)$ is a system

$$
\Omega_{g, n} \in H^{*}\left(\bar{M}_{g, n}\right) \otimes\left(V^{*}\right)^{\otimes n}
$$

of multilinear forms with values in the cohomology ring of $\bar{M}_{g, n}$ satisfying the following properties:

$S_{n}$-Equivariance $\Omega_{g, n}$ is $S_{n}$-equivariant with respect to the $S_{n}$-action permuting the markings of $\bar{M}_{g, n}$ and the factors of $\left(V^{*}\right)^{\otimes n}$.

Gluing The pull-back of $\Omega_{g, n}$ via the gluing map

$$
\bar{M}_{g_{1}, n_{1}+1} \times \bar{M}_{g_{2}, n_{2}+1} \rightarrow \bar{M}_{g, n}
$$

is given by the direct product of $\Omega_{g_{1}, n_{2}+1}$ and $\Omega_{g_{2}, n_{2}+1}$ with the bivector $\eta^{-1}$ inserted at the two points glued together. Similarly, for the gluing map $\bar{M}_{g-1, n+2} \rightarrow \bar{M}_{g, n}$, the pull-back of $\Omega_{g, n}$ is given by $\Omega_{g-1, n+2}$ with $\eta^{-1}$ inserted at the two points glued together.

Unit There is a special element $\mathbf{1} \in V$ called the unit such that

$$
\Omega_{g, n+1}\left(v_{1}, \ldots, v_{n}, \mathbf{1}\right)
$$

is the pull-back of $\Omega_{g, n}\left(v_{1}, \ldots, v_{n}\right)$ under the forgetful map and

$$
\Omega_{0,3}(v, w, \mathbf{1})=\eta(v, w) .
$$

Definition 3.1.2. A CohFT which takes values in $H^{0}$ (so that each $\Omega_{g, n}$ is a multiple of the fundamental class) is called a topological field theory (TQFT).

The definition of CohFTs can be also generalized to families of CohFTs over a ground ring. We will use the following non-standard definition.

Definition 3.1.3. Let $e_{1}, \ldots, e_{N}$ be a basis of $V$. A convergent CohFT $\Omega$ on $V$ is a CohFT defined over the ring of holomorphic functions of an open neighborhood $U$ of $0 \in V$ such that for all $g \geq 0$, all $\alpha_{1}, \ldots, \alpha_{n} \in V$ and all $\mathbf{t}=t_{1} e_{1}+\cdots+t_{N} e_{N} \in U$ we have

$$
\left.\Omega_{g, n}\right|_{\mathbf{t}}\left(\alpha_{1}, \ldots, \alpha_{n}\right)=\left.\sum_{k=0}^{\infty} \frac{1}{k !} \pi_{*} \Omega_{g, n+k}\right|_{0}\left(\alpha_{1}, \ldots, \alpha_{n}, \mathbf{t}, \ldots, \mathbf{t}\right),
$$

where $\pi: \bar{M}_{g, n+k} \rightarrow \bar{M}_{g, n}$ forgets the last $k$ markings.

Remark 3.1.4. If $\Omega$ is defined via the Gromov-Witten theory of $X$, then convergence of $\Omega$ implies convergence of the total ancestor potential of $X$ in the sense of [3. This follows immediately by integration of $\Omega_{g, n}$ over $\bar{M}_{g, n}$ against monomials in $\psi$-classes. A priori, the integrals of $\Omega$ against $\psi$-classes contain less information than $\Omega$ itself, so it is not clear whether the convergence of the CohFT is equivalent to the convergence of its ancestor potential. Still, it should be straightforward to adapt many proofs of convergence of the ancestor potential to show convergence of the corresponding CohFT level (see also Section 3.8). 
We can define from any usual CohFT a convergent CohFT by using (2), under the assumption that the sum converges in a neighborhood of 0 .

Definition 3.1.5. The underlying Frobenius manifold of a convergent CohFT $\Omega$ is, as a manifold, the neighborhood $U$ of $0 \in V$. At every point $p$ of $U$, the tangent space is identified with $V$ via the map sending $\frac{\partial}{\partial t_{\mu}}$ at $p$ to $e_{\mu}$. With this identification, $\eta$ defines the metric, 1 defines the unit vector field and $\Omega_{0,3}$ defines the symmetric tensor $A$.

Remark 3.1.6. Restricting to the origin, we see that every CohFT determines a Frobenius algebra. This operation restricts to a bijection between TQFTs and Frobenius algebras of dimension $N$.

Using the underlying Frobenius manifold, for any convergent CohFT we can define the quantum product on $V$ (depending on a point in $U$ ), semisimplicity and the discriminant locus.

Example 3.1.7. Given an $N$-dimensional (convergent) CohFT $\Omega$ and some $c \in \mathbb{C}^{*}$, we can define an $(N+1)$-dimensional (convergent) CohFT $\Omega^{\prime}:$ If $V$ is the underlying vector space of $\Omega$, then $V \oplus\langle v\rangle$ will be the underlying vector space of $\Omega^{\prime}$. The nonsingular bilinear form $\eta^{\prime}$ on $V \oplus \mathbb{C}$ is defined via $\eta^{\prime}(v, v)=c$, $\eta^{\prime}(\alpha, v)=0$ and $\eta^{\prime}(\alpha, \beta)=\eta(\alpha, \beta)$, where $\alpha, \beta \in V$ and $\eta$ is the nonsingular bilinear form of $V$. The CohFT $\Omega^{\prime}$ is then defined by multilinearity from setting

$$
\Omega_{g, n}^{\prime}\left(\alpha_{1}, \ldots, \alpha_{n}\right)=\Omega_{g, n}\left(\alpha_{1}, \ldots, \alpha_{n}\right),
$$

if all $\alpha_{i}$ lie in $V$, imposing the condition that $\Omega^{\prime}$ vanishes if one argument is a multiple of $v$ and another argument lies in $V$, and setting

$$
\Omega_{g, n}^{\prime}(v, \ldots, v)=c^{1-g} .
$$

Finding the right definition in the remaining case $n=0$ and checking the axioms of a CohFT is left as an exercise to the reader.

Notice that $v$ will be an idempotent element for the quantum product and that this operation therefore preserves semisimplicity.

Definition 3.1.8. A convergent CohFT $\Omega_{g, n}$ is called homogeneous if its underlying Frobenius manifold is conformal and the CohFT is an eigenvector of $L_{E}$ of eigenvalue $(g-1) \delta+n$.

As the name suggests, a convergent CohFT is homogeneous if it carries a grading such that all natural structures are homogeneous with respect to the grading.

\subsection{Reconstruction}

The (upper half of the) symplectic loop group corresponding to a vector space $V$ with nonsingular bilinear form $\eta$ is the group of endomorphism valued power series $V \llbracket z \rrbracket$ such that the symplectic condition $R(z) R^{t}(-z)=1$ holds. Here $R^{t}$ 
is the adjoint of $R$ with respect to $\eta$. There is an action of this group on the space of all CohFTs based on a fixed semisimple Frobenius algebra structure of $V$. The action is often named after Givental because he has introduced it on the level of arbitrary genus Gromov-Witten potentials.

Given a CohFT $\Omega_{g, n}$ and such an endomorphism $R$, the new CohFT $R \Omega_{g, n}$ takes the form of a sum over dual graphs $\Gamma$

$$
R \Omega_{g, n}\left(v_{1}, \ldots, v_{n}\right)=\sum_{\Gamma} \frac{1}{\operatorname{Aut}(\Gamma)} \xi_{*}\left(\prod_{v} \sum_{k=0}^{\infty} \frac{1}{k !} \pi_{*} \Omega_{g_{v}, n_{v}+k}(\ldots)\right)
$$

where $\xi: \prod_{v} \bar{M}_{g_{v}, n_{v}} \rightarrow \bar{M}_{g, n}$ is the gluing map of curves of topological type $\Gamma$ from their irreducible components, $\pi: \bar{M}_{g_{v}, n_{v}+k} \rightarrow \bar{M}_{g_{v}, n_{v}}$ forgets the last $k$ markings and we still need to specify what is put into the arguments of $\prod_{v} \Omega_{g_{v}, n_{v}+k}$. Instead of only allowing vectors in $V$ to be put into $\Omega_{g, n}$ we will also allow for elements of $V \llbracket \psi_{1}, \ldots, \psi_{n} \rrbracket$ where $\psi_{i}$ acts on the cohomology of the moduli space by multiplication with the $i$ th cotangent line class.

- Into each argument corresponding to a marking of the curve, put $R^{-1}(\psi)$ applied to the corresponding vector.

- Into each pair of arguments corresponding to an edge put the bivector

$$
\frac{R^{-1}\left(\psi_{1}\right) \otimes R^{-1}\left(\psi_{2}\right)-\mathrm{Id}}{-\psi_{1}-\psi_{2}} \eta^{-1} \in V^{\otimes 2} \llbracket \psi_{1}, \psi_{2} \rrbracket,
$$

where one has to substitute the $\psi$-classes at each side of the normalization of the node for $\psi_{1}$ and $\psi_{2}$. By the symplectic condition this is well-defined.

- At each of the additional arguments for each vertex put

$$
T(\psi):=\psi\left(\mathrm{Id}-R^{-1}(\psi)\right) \mathbf{1},
$$

where $\psi$ is the cotangent line class corresponding to that vertex. Since $T(z)=O\left(z^{2}\right)$ the above $k$-sum is finite.

The following reconstruction result (on the level of potentials) has been first proposed by Givental [7].

Theorem 3.2.1 ([24]). The R-matrix action is free and transitive on the space of semisimple CohFTs based on a given Frobenius algebra.

Furthermore, given a convergent semisimple CohFT $\Omega$, locally around a semisimple point, the element $R$ of the symplectic loop group, taking the TQFT corresponding to the Frobenius algebra to $\Omega$, satisfies the following differential equation of one-forms when written in a basis of normalized idempotents

$$
[R(z), d \mathbf{u}]+z \Psi^{-1} d(\Psi R(z))=0 .
$$

Here $\mathbf{u}$ is the diagonal matrix filled with the canonical coordinates $u_{i}$ corresponding to the idempotents and $\Psi$ is the basis change from the basis of normalized idempotents to a flat basis. 
Remark 3.2.2. The differential equation (4) makes sense for any Frobenius manifold. In general it defines $R$ only up to right multiplication by a diagonal matrix whose entries are of the form $\exp \left(a_{1} z+a_{3} z^{3}+\cdots\right)$, where the $a_{i}$ are constant on the Frobenius manifold 8 . If the further condition of homogeneity with respect to an Euler vector field is imposed on $R$, there is a unique solution.

Remark 3.2.3. Teleman's proof relies heavily on topological results (Mumford's conjecture/Madsen-Weiss theorem) and it is therefore not known if the same classification result also holds in general when we work in Chow instead of cohomology. It is still known that the Chow-valued statement holds in some cases such as for the equivariant Gromov-Witten theory of a toric variety.

Remark 3.2.4. Formula (1) for a genus one potential

$$
\mathrm{d} G(X)=\int_{\bar{M}_{1,1}} \Omega_{1,1}(X)
$$

is a special case of the reconstruction.

For later use, let us use the normalized idempotent basis to make the reconstruction formula a bit more concrete: We can decompose any endomorphism $F$ of $V$ into a collection of linear forms

$$
F=\sum_{i} F^{i} \tilde{\epsilon}_{i},
$$

where $\tilde{\epsilon}_{i}$ is the $i$ th normalized idempotent element, and we will use the formula

$$
\omega_{g, n}\left(\tilde{\epsilon}_{a_{1}}, \ldots, \tilde{\epsilon}_{a_{n}}\right)= \begin{cases}\sum_{i} \Delta_{i}^{g-1}, & \text { if } n=0, \\ \Delta_{a_{1}}^{\frac{2 g-2+n}{2}}, & \text { if } a_{1}=\cdots=a_{n}, \\ 0, & \text { else, }\end{cases}
$$

where the $\Delta_{i}$ are the inverses of the norms of the idempotents. Then we can rewrite (3) to

$$
R \Omega_{g, n}\left(v_{1}, \ldots, v_{n}\right)=\sum_{\Gamma, c} \frac{1}{\operatorname{Aut}(\Gamma, c)} \xi_{*}\left(\prod_{v} C_{v, c(v)}(\ldots)\right),
$$

where $c$ is a coloring of the vertices of $\Gamma$ by a color in the set $\{1, \ldots, N\}$, and the local contribution $C_{v, i}$ at a vertex $v$ of genus $g$, with $n$ markings and of color $i$ is an $n$-form taking power series in $z$ as inputs, and is given by

$$
\begin{aligned}
& C_{v, i}\left(\alpha_{1}, \ldots, \alpha_{n}\right) \\
& =\sum_{k=0}^{\infty} \frac{\Delta_{i}^{\frac{2 g-2+n+k}{2}}}{k !} \pi_{*}\left(\prod_{j=1}^{n} \alpha_{j}\left(\psi_{j}\right) \prod_{j=n+1}^{n+k} \psi_{j}\left(\operatorname{Id}^{i}-\left(R^{-1}\left(\psi_{j}\right)\right)^{i}\right) \mathbf{1}_{\Omega}\right) .
\end{aligned}
$$

The still missing arguments in (5), which correspond to preimages of the marked points and nodes in the normalization, are the same as the vectors and bivectors also used in (3), except that we need to take their coordinates as prescribed by the coloring. 


\subsection{Relations in the tautological ring}

The tautological subrings $R^{*}\left(\bar{M}_{g, n}\right)$ can be compactly defined [5] as the smallest system of subrings $R^{*}\left(\bar{M}_{g, n}\right) \subseteq H^{*}\left(\bar{M}_{g, n}\right)$ stable under push-forward under the tautological maps as described in Section 3.1. Each tautological ring has a finite additive generating set [9] which is indexed by strata of $\bar{M}_{g, n}$ (corresponding to dual graphs) decorated with Morita-Mumford-Miller $\kappa$-classes and $\psi$-classes. The formal algebra generated by these generators is called the strata algebra $\mathcal{S}_{g, n}$ [23]. Pushforwards and pullbacks along the gluing and forgetful morphisms can be lifted to homomorphisms of the corresponding strata algebras satisfying the push-pull formula, .... Relations in the tautological ring are elements of the kernel of the natural projection $q: \mathcal{S}_{g, n} \rightarrow R^{*}\left(\bar{M}_{g, n}\right)$.

Consider a semisimple, $N$-dimensional convergent CohFT $\Omega$ defined in a neighborhood $U$ of $0 \in V$. Let $D \subset U$ be the discriminant locus. By the reconstruction formula described in Section 3.2 , for each point outside $D$ in $U$ we can find an $R$-matrix such that $\Omega$ is given by applying the action of $R$ to the underlying TQFT.

We obtain relations in the tautological ring by studying the behavior along $D$. On the one hand, the reconstruction formula defines a function $\widetilde{\Omega}_{g, n} \in \mathcal{S}_{a, n} \otimes$ $\left(V^{*}\right)^{\otimes n} \otimes \mathcal{O}_{U \backslash D}$ that might have singularities along the discriminant locus 4 On the other hand, by Theorem 3.2.1, we recover the original CohFT if we project from the strata algebra to the tautological ring, so that $q\left(\widetilde{\Omega}_{g, n}\right) \in R^{*}\left(\bar{M}_{g, n}\right) \otimes$ $\left(V^{*}\right)^{\otimes n} \otimes \mathcal{O}_{U}$. Therefore, if $p: \mathcal{O}_{U \backslash D} \rightarrow\left(\mathcal{O}_{U \backslash D} / \mathcal{O}_{U}\right)$ denotes the projection, then $p\left(\widetilde{\Omega}_{g, n}\right) \in \operatorname{ker}(q) \otimes\left(V^{*}\right)^{\otimes n} \otimes\left(\mathcal{O}_{U \backslash D} / \mathcal{O}_{U}\right)$ is a $\left(V^{*}\right)^{\otimes n} \otimes\left(\mathcal{O}_{U \backslash D} / \mathcal{O}_{U}\right)$-valued tautological relation, from which we can extract many ordinary tautological relations by applying linear forms $\varphi:\left(V^{*}\right)^{\otimes n} \otimes\left(\mathcal{O}_{U \backslash D} / \mathcal{O}_{U}\right) \rightarrow \mathbb{C}$.

Definition 3.3.1. The vector space of tautological relations associated to the convergent CohFT $\Omega$ is defined as the smallest system of ideals of $\mathcal{S}_{g, n}$ which is stable under push-forwards via the gluing and forgetful morphisms and contains the relations $p\left(\widetilde{\Omega}_{g, n}\right)$ from cancellations of singularities in the reconstruction of $\Omega$, that we have just discussed.

Example 3.3.2. For the 2-dimensional, (convergent) CohFT corresponding to Witten's 3-spin class, in [20] it is proven that the ideal of relations coincides with the relations of Pixton [23, which are conjectured to be all relations between tautological classes.

Example 3.3.3. In 14 it is shown that the ideal of relations of the GromovWitten theory of equivariant projective space $\mathbb{P}^{N-1}$ contains the relations for Witten's $(N+1)$-spin class.

Example 3.3.4. In 14 it is also shown that the set of relations for equivariant $\mathbb{P}^{1}$ and Witten's 3-spin class coincide.

\footnotetext{
${ }^{4}$ The proof of Theorem 3.3.6 will imply that for a CohFT the only possible singularities of the $R$-matrix along $D$ are poles.
} 
Remark 3.3.5. For nonequivariant $\mathbb{P}^{1}$, the theory does not apply since the Frobenius manifold is semisimple at all points. There is however a different way of how to extract relations in this case, which we will study in Section 3.8.

The following is our main result.

Theorem 3.3.6. For any two semisimple convergent CohFTs which are not semisimple at all points of the underlying Frobenius manifold, the sets of associated tautological relations coincide.

Remark 3.3.7. In the proof of Theorem 3.3.6 we will first, locally near a smooth point on the discriminant, identify canonical coordinates and normalized idempotents. An important part of the proof is to show that under this identification the quotient of corresponding $R$-matrices is holomorphic along the discriminant.

In 14] for the comparison of equivariant $\mathbb{P}^{1}$ and the $A_{2}$-singularity a different, more explicit identification of coordinates and vector fields is chosen. Therefore, while with this identification the quotient of the $R$-matrices is not holomorphic along the discriminant, there exists a holomorphic function $\varphi$ such that $R_{\mathbb{P}^{1}}(z)=R(z) R_{A_{2}}(\varphi z)$. This result depends on the fact that the $A_{2}$-theory is homogeneous.

Remark 3.3.8. The more classical way of 20] and [21] to obtain tautological relations from a convergent CohFT works by considering cohomological degrees: Assume that $\Omega$ is in addition homogenous for an Euler vector field $E$, that all $\beta_{i}$ vanish and that all $\alpha_{i}$ as in Definition 2.1.2 are positive. Then the homogeneity implies that the cohomological degree of $\Omega_{g, n}\left(\frac{\partial}{\partial t_{a_{1}}}, \ldots, \frac{\partial}{\partial t_{a_{n}}}\right)$ is bounded by

$$
(g-1) \delta+n-\sum_{j} \alpha_{a_{j}}
$$

However the reconstructed theory might also contain terms of higher cohomological degree. These thus have to vanish, giving tautological relations. To make them more concrete, the coefficients of these relations, which are functions on the underlying Frobenius manifold, can be evaluated at any point outside of the discriminat.

These relations from degree considerations follow from the relations of Definition 3.3.1. To see this, notice that by positivity of the $\alpha_{i}$, the action of $E$ on

holomorphic functions on the underlying Frobenius manifold has only positive eigenvalues. Therefore and because of homogeneity (and generic semisimplicity), coefficients of the part of the reconstruction formula violating the bound (6) in particular cannot be holomorphic along the discriminant.

\subsection{Local extension}

The proof of the following theorem will occupy this section. The content of the proof is also used for proving Theorem 3.3.6.

Theorem 3.4.1. Let $M$ be an $N$-dimensional semisimple Frobenius manifold and let $p$ be a smooth point of the discriminant of $M$ such that $m=3$ in 
Theorem 2.2.2, Then, after possibly shrinking $M$ to a smaller neighborhood of $p$, there exists a convergent CohFT with underlying Frobenius manifold $M$.

We first study the consequences of Theorem 2.2.2 in more detail. After possibly shrinking $M$, it gives us a basis of holomorphic vector fields $\left\{\frac{\partial}{\partial t_{0}}, \frac{\partial}{\partial t}, \frac{\partial}{\partial u_{\geq 3}}\right\}$, where

$$
\frac{\partial}{\partial t_{0}}=\frac{\partial}{\partial u_{1}}+\frac{\partial}{\partial u_{2}}, \quad \frac{\partial}{\partial t}=\left(\frac{3}{4}\left(u_{1}-u_{2}\right)\right)^{\frac{1}{3}}\left(\frac{\partial}{\partial u_{1}}-\frac{\partial}{\partial u_{2}}\right) .
$$

It is easy to see that these vector fields commute and therefore we can integrate them to coordinates $t_{0}, t$ and $u_{\geq 3}$. The discriminant locus $D$ is then locally given by the equation $t=0$.

Notice that there is a root $\sqrt{t}$ of $t$ such that

$$
\frac{\partial}{\partial t}=\sqrt{t}\left(\frac{\partial}{\partial u_{1}}-\frac{\partial}{\partial u_{2}}\right), \quad\left(\frac{\partial}{\partial t}\right)^{2}=t\left(\frac{\partial}{\partial u_{1}}+\frac{\partial}{\partial u_{2}}\right) .
$$

Define holomorphic functions $\eta_{0}$ and $\eta_{1}$ by

$$
\eta_{0}=\eta\left(\frac{\partial}{\partial t_{0}}, \frac{\partial}{\partial t_{0}}\right), \quad \eta_{1}=\eta\left(\frac{\partial}{\partial t}, \frac{\partial}{\partial t_{0}}\right)
$$

and notice that

$$
\eta\left(\frac{\partial}{\partial t}, \frac{\partial}{\partial t}\right)=\eta\left(\frac{\partial}{\partial t} \star \frac{\partial}{\partial t}, \sum_{i=1}^{N} \frac{\partial}{\partial u_{i}}\right)=t \eta_{0} .
$$

Since $\eta$ is nonsingular, $\eta_{1}$ cannot vanish on the discriminant. The inverses $\Delta_{1}$, $\Delta_{2}$ of the norms of the first two idempotents are given by

$$
\Delta_{1}=\frac{2 \sqrt{t}}{\eta_{1}+\sqrt{t} \eta_{0}}, \quad \Delta_{2}=\frac{-2 \sqrt{t}}{\eta_{1}-\sqrt{t} \eta_{0}} .
$$

We next choose roots $\sqrt{2 \sqrt{t}}, \sqrt{-2 \sqrt{t}}$ and $\sqrt{\eta_{1}}$. These induce roots of $\Delta_{1}$, $\Delta_{2}$, which we will use to define the normalized idempotents. Let $\Psi_{0}$ be the block diagonal matrix with upper left block being

$$
\left(\begin{array}{cc}
\frac{\sqrt{t}}{\sqrt{2 \sqrt{t}}} & \frac{-\sqrt{t}}{\sqrt{-2 \sqrt{t}}} \\
\frac{1}{\sqrt{2 \sqrt{t}}} & \frac{1}{\sqrt{-2 \sqrt{t}}}
\end{array}\right)
$$

and the identity matrix as the lower right block. For $\Psi_{0}^{-1}$ the upper left block is given by

$$
\left(\begin{array}{cc}
\frac{1}{\sqrt{2 \sqrt{t}}} & \frac{\sqrt{t}}{\sqrt{2 \sqrt{t}}} \\
\frac{1}{\sqrt{-2 \sqrt{t}}} & \frac{-\sqrt{t}}{\sqrt{-2 \sqrt{t}}}
\end{array}\right) .
$$

The matrix $\Psi_{0}$ is the basis change from normalized idempotents to the basis $\left\{\frac{\partial}{\partial t_{0}}, \frac{\partial}{\partial t}, \frac{\partial}{\partial u_{\geq 3}}\right\}$. In the $A_{2}$-singularity case $\eta_{0}=0, \sqrt{\eta_{1}}=1$ and $\sqrt{\Delta_{\geq 3}}=1$.

Let $\Psi_{1}$ denote the basis change from the normalized idempotent basis to a flat basis and define $\tilde{\Psi}_{1}=\Psi_{1} \Psi_{0}^{-1}$. 
Lemma 3.4.2. The basis change matrix $\tilde{\Psi}_{1}$ is holomorphic along D.

Proof. By Theorem 2.2 .2 it is enough to prove the same statement for $\tilde{\Psi}^{\prime}:=$ $\Psi^{\prime} \Psi_{0}^{-1}$ where $\Psi^{\prime}$ is the basis change from the normalized idempotent basis to the basis $\left\{\frac{\partial}{\partial t_{0}}, \frac{\partial}{\partial t}, \frac{\partial}{\partial u_{\geq 3}}\right\}$. Since the basis changes leave all but the first two idempotents invariant we will only need to consider the upper-left $2 \times 2$ block of $\tilde{\Psi}^{\prime}$. We factor this block into

$$
\left(\begin{array}{cc}
\frac{\sqrt{t}}{2 \sqrt{t}} & \frac{-\sqrt{t}}{-2 \sqrt{t}} \\
\frac{1}{2 \sqrt{t}} & \frac{1}{-2 \sqrt{t}}
\end{array}\right)\left(\begin{array}{cc}
\frac{\sqrt{\Delta_{1}}}{\sqrt{2 t}} & 0 \\
0 & \frac{\sqrt{\Delta_{2}}}{\sqrt{-2 t}}
\end{array}\right)\left(\begin{array}{cc}
1 & \sqrt{t} \\
1 & -\sqrt{t}
\end{array}\right),
$$

a change from $\left\{\frac{\partial}{\partial t_{0}}, \frac{\partial}{\partial t}\right\}$ to the idempotents, a multiplication by a diagonal matrix and the change back from the idempotents to $\left\{\frac{\partial}{\partial t_{0}}, \frac{\partial}{\partial t}\right\}$. So we see that the upper left block of $\tilde{\Psi}^{\prime}$ has the form

$$
\eta_{1}^{-\frac{1}{2}}\left(\begin{array}{cc}
a & t c \\
c & a
\end{array}\right)
$$

where

$$
\begin{gathered}
a=\frac{1}{2}\left(\frac{1}{\sqrt{1+\sqrt{t} \frac{\eta_{0}}{\eta_{1}}}}+\frac{1}{\sqrt{1-\sqrt{t} \frac{\eta_{0}}{\eta_{1}}}}\right)=1+\frac{3}{8} \frac{\eta_{0}^{2}}{\eta_{1}^{2}} t+O\left(t^{2}\right) \\
c=\frac{1}{2 \sqrt{t}}\left(\frac{1}{\sqrt{1+\sqrt{t} \frac{\eta_{0}}{\eta_{1}}}}-\frac{1}{\sqrt{1-\sqrt{t} \frac{\eta_{0}}{\eta_{1}}}}\right)=-\frac{1}{2} \frac{\eta_{0}}{\eta_{1}}-\frac{5}{16} \frac{\eta_{0}^{3}}{\eta_{1}^{3}} t+O\left(t^{2}\right)
\end{gathered}
$$

which are holomorphic along the discriminant.

We can repeat this setup for any other $N$-dimensional Frobenius manifold satisfying the assumptions of Theorem 3.4.1, in particular, as we will assume from now on, for any Frobenius manifold $M^{2}$ underlying an $N$-dimensional convergent CohFT $\Omega^{2}$, such as an extension of the theory of the $A_{2}$-singularity to $N$ dimensions using the construction of Example 3.1.7 repeatedly. Our main strategy in order to prove Theorem 3.4.1 is to show that a local extension $\Omega^{1}$ can be obtained from $\Omega^{2}$ by the action of a holomorphic $R$-matrix and a holomorphic shift.

For this, we first use the coordinates $t, t_{0}, u_{>3}$ to identify a small neighborhood of $p$ with a small neighborhood of the origin of $M^{2}$. Let us shrink $M$ accordingly. Notice that this isomorphism of complex manifolds, if the third roots in (7) have been chosen compatibly, amounts, outside of the discriminant, to identifying their canonical coordinates. We accordingly identify normalized idempotents and thereby the tangent spaces that they span. This identification preserves the metric but not the quantum product structure. In particular, the basis change $\Psi_{2}$ from the normalized idempotent basis to a basis of flat vector fields on $M^{2}$ in general does not agree with $\Psi_{1}$. We set $\tilde{\Psi}_{2}=\Psi_{2} \Psi_{0}^{-1}$, which by Lemma 3.4 .2 is also holomorphic along $D$. 
The following lemma constructs the holomorphic $R$-matrix we will use in the construction of $\Omega^{1}$ from $\Omega^{2}$.

Lemma 3.4.3. There exists a symplectic solution $R_{1}$ of the flatness equation (44) for $\Psi_{1}$ such that if $R_{2}$ denotes the solution of (44) for $\Psi_{2}$ used for reconstructing the CohFT $\Omega^{2}$, the endomorphism $R_{1} R_{2}^{-1}$ is holomorphic (under the identifications we have made above).

Proof. For this proof let $R_{1}$ and $R_{2}$ denote the $R$-matrices written in the basis of normalized idempotents instead of the underlying endomorphism valued power series. We set

$$
R_{i}=\Psi_{0}^{-1} \tilde{R}_{i} \Psi_{0}
$$

where $\Psi_{0}$ is as in (8). We can write the flatness equations (4) as

$$
\left[\tilde{R}_{i}, \Psi_{0} \mathrm{~d} u \Psi_{0}^{-1}\right]+z \tilde{\Psi}_{i}^{-1} \mathrm{~d}\left(\tilde{\Psi}_{i} \tilde{R}_{i} \Psi_{0}\right) \Psi_{0}^{-1}=0 .
$$

If $R:=\tilde{R}_{1} \tilde{R}_{2}^{-1}$, these two differential equations combine to

$$
0=\left[R, \Psi_{0} \mathrm{~d} u \Psi_{0}^{-1}\right]+z \tilde{\Psi}_{1}^{-1} \mathrm{~d}\left(\tilde{\Psi}_{1} R \tilde{\Psi}_{2}^{-1}\right) \tilde{\Psi}_{2} .
$$

By Lemma 3.4.2 it is enough to show that there is a solution $R$ of (10) all of whose entries are holomorphic along the discriminant and which satisfies the symplectic condition.

We analyze the entries of the ingredients in (10). For this we will consider all matrices to consist of four blocks numbered according to

$$
\left(\begin{array}{ll}
1 . & 2 . \\
3 . & 4 .
\end{array}\right)
$$

such that the first block has size $2 \times 2$. By Lemma 3.4 .2 the matrices $\tilde{\Psi}_{i}^{-1}, \tilde{\Psi}_{i}$ for $i \in\{1,2\}$ and therefore also the matrices $\tilde{\Psi}_{1}^{-1} \mathrm{~d} \tilde{\Psi}_{1}$ and $\left(\mathrm{d} \tilde{\Psi}_{2}^{-1}\right) \tilde{\Psi}_{2}$ of one-forms are holomorphic along $D$. The matrix $\left(\mathrm{d} \Psi_{0}\right) \Psi_{0}^{-1}$ has all blocks equal to zero except for the first one, which is

$$
\frac{\mathrm{d} t}{4 t}\left(\begin{array}{cc}
1 & 0 \\
0 & -1
\end{array}\right)
$$

and the matrix $\Psi_{0} \mathrm{~d} \mathbf{u} \Psi_{0}^{-1}$ is block diagonal with first block being

$$
\left(\begin{array}{cc}
\mathrm{d} t_{0} & t \mathrm{~d} t \\
\mathrm{~d} t & \mathrm{~d} t_{0}
\end{array}\right)
$$

and the other block being the diagonal matrix with entries $\mathrm{d} u_{\geq 3}$. Furthermore, because in general $\Psi_{1}^{-1} \mathrm{~d} \Psi_{1}$ is antisymmetric (as can be seen by differentiating $\left.\Psi_{1}^{t} \eta \Psi_{1}=1\right)$ and from (8), (9) and (11) we see that the first block of $\tilde{\Psi}_{1}^{-1} \mathrm{~d} \tilde{\Psi}_{1}$ has the general form

$$
\left(\begin{array}{cc}
x & 0 \\
0 & -x
\end{array}\right)
$$


and the forth block is still antisymmetric. The same holds for $\left(\mathrm{d} \tilde{\Psi}_{2}^{-1}\right) \tilde{\Psi}_{2}$.

We construct the coefficients of $R$ inductively. Let us set

$$
R(z)=\sum_{i=0}^{\infty} R^{i} z^{i}
$$

and $R_{j k}^{i}$ for the entries of $R^{i}$. We assume that we have already constructed $R^{j}$ for $j \leq i$ satisfying the flatness equation and symplectic condition modulo $z^{i+1}$.

Because of (12), inserting $\frac{\partial}{\partial t_{0}}$ into the $z^{i+1}$-part of (10) directly gives equations for the off-diagonal blocks of $R^{i+1}$ in terms of holomorphic functions.

Similarly, inserting $\frac{\partial}{\partial u_{\geq 3}}$ into the $z^{i+1}$-part of (10) gives us holomorphic formulas for the off-diagonal entries of $R^{i+1}$ in the forth block. For the diagonal entries of this block we instead insert $\frac{\partial}{\partial t}$ into the $z^{i+2}$-part of (10) and because of the antisymmetry obtain that the first $t$-derivatives of the diagonal entries are holomorphic. We can integrate them locally and have an arbitrary choice of integration constants (ignoring the symplectic condition for the moment).

It remains the analysis of the first block. For this it is useful to compute the commutator

$$
\left[\left(\begin{array}{ll}
R_{11}^{i+1} & R_{12}^{i+1} \\
R_{21}^{i+1} & R_{22}^{i+1}
\end{array}\right),\left(\begin{array}{ll}
0 & t \\
1 & 0
\end{array}\right)\right]=\left(\begin{array}{cc}
R_{12}^{i+1}-t R_{21}^{i+1} & t\left(R_{11}^{i+1}-R_{22}^{i+1}\right) \\
R_{22}^{i+1}-R_{11}^{i+1} & t R_{21}^{i+1}-R_{12}^{i+1}
\end{array}\right)
$$

So the insertion of $\frac{\partial}{\partial t}$ into the $z^{i+1}$-part of (10) gives holomorphic formulas for $R_{11}^{i+1}-R_{22}^{i+1}$ and $R_{12}^{i+1}-t R_{21}^{i+1}$. Because of (11) and (13) the $\frac{\partial}{\partial t}$ insertion into the $z^{i+2}$-part of (10) shows that the first $t$-derivative of $R_{11}^{i+1}+R_{22}^{i+1}$ is holomorphic and therefore this sum is holomorphic and we again have the choice of an integration constant. Similarly, we find that

$$
2 t \frac{\partial}{\partial t} R_{21}^{i+1}+R_{21}^{i+1}
$$

is holomorphic in $D$ and therefore $R_{21}^{i+1}$ is holomorphic up to a possible constant multiple of $t^{-\frac{1}{2}}$. Here, we have a unique choice of integration constant giving a holomorphic solution.

In general the symplectic condition does not constrain the integration constants of $R^{i+1}$ when $i+1$ is odd 8 . On the other hand, it completely determines the integration constants of $R^{i+1}$ when $i+1$ is even. It is clear that the solution determined by the symplectic condition is meromorphic, and hence by the above analysis, it is also holomorphic.

Let $R_{1}$ and $R_{2}$ be as in the lemma. We can then define a convergent CohFT $\Omega^{1}$ defined outside the discriminant locus by the $R$-matrix action of $R_{1}$ on the trivial CohFT. We will show that $\Omega^{1}$ extends to the discriminant locus by comparing it to the (everywhere defined) convergent CohFT $\Omega^{3}$ defined by the $R$-matrix action $\Omega^{3}=\left(R_{1} R_{2}^{-1}\right) \Omega^{2}$. The CohFTs $\Omega^{3}$ and $\Omega^{1}$ are very similar but the underlying trivial theories do not agree, in particular the units $\mathbf{1}_{\Omega^{1}}$ and 
$\mathbf{1}_{\Omega^{2}}$ as well as the norms $\Delta_{1 i}^{-1}$ and $\Delta_{2 i}^{-1}$ of the idempotents are different. We will fix this using a shift.

For this, recall the description (5) of the reconstruction using the basis of normalized idempotents. A local contribution at a vertex of color $i$ for the reconstruction of $\Omega^{3}$ is of the form

$$
\Delta_{2 i}^{\frac{2 g-2+n}{2}} \sum_{k=0}^{\infty} \frac{\Delta_{2 i}^{\frac{k}{2}}}{k !} \pi_{*}\left(\prod_{j=1}^{n} \alpha_{j}^{i} \prod_{j=1}^{k} \psi_{j}\left(\operatorname{Id}^{i}-\left(R_{1}^{-1}\left(\psi_{j}\right)\right)^{i}\right) \mathbf{1}_{\Omega^{2}}\right)
$$

where $\pi$ forgets the last $k$ markings and $\alpha_{j}^{i}$ are some formal series in $\psi_{j}$ whose coefficients are holomorphic functions on the Frobenius manifold. To circumvent convergence issues, let $v$ be a formal flat vector field and let $v_{\mu}$ and $v_{i}$ be the coordinates of $v$ when written in a basis of flat coordinates or in the basis of normalized idempotents, respectively. We can further modify the CohFT by shifting along $v \psi$ :

$$
\Omega_{g, n}^{4}\left(\alpha_{1}, \ldots, \alpha_{n}\right):=\sum_{k=0}^{\infty} \frac{1}{k !} \pi_{*} \Omega_{g, n+k}^{3}\left(\alpha_{1}, \ldots, \alpha_{n}, \psi v, \ldots, \psi v\right)
$$

We obtain a well-defined convergent CohFT defined over the ring of power series in the $v_{\mu}$. For $\Omega^{4}$ the local contribution at a vertex of color $i$ is

$$
\Delta_{2 i}^{\frac{2 g-2+n}{2}} \sum_{k=0}^{\infty} \frac{\Delta_{2 i}^{\frac{k}{2}}}{k !} \pi_{*}\left(\prod_{j=1}^{n} \alpha_{j}^{i} \prod_{j=1}^{k} \psi_{j}\left[\operatorname{Id}^{i} \mathbf{1}_{\Omega^{2}}-\left(R_{1}^{-1}\left(\psi_{j}\right)\right)^{i}\left(\mathbf{1}_{\Omega^{2}}-v\right)\right]\right) .
$$

Recall that the dilaton equation implies that

$$
\frac{1}{(1-a)^{2 g-2+n}}=\sum_{k=0}^{\infty} \frac{1}{k !} \pi_{*}\left(\prod_{j=1}^{k} a \psi_{j}\right)
$$

where $a$ is a formal variable, or equivalently

$$
1=\sum_{k=0}^{\infty} \frac{(1+b)^{2-2 g-n-k}}{k !} \pi_{*}\left(\prod_{j=1}^{k} b \psi_{j}\right)
$$

where $b=a /(1-a)$. We will apply this identity locally at every vertex. At a vertex of color $i$ we use $-\sqrt{\Delta_{2 i}} v_{i}$ for $b$. Then the local contribution at a vertex of color $i$ is

$$
\Delta_{3 i}^{\frac{2 g-2+n}{2}} \sum_{k=0}^{\infty} \frac{\Delta_{3 i}^{\frac{k}{2}}}{k !} \pi_{*}\left(\prod_{j=1}^{n} \alpha_{j}^{i} \prod_{j=1}^{k} \psi_{j}\left[\operatorname{Id}^{i}-\left(R_{1}^{-1}\left(\psi_{j}\right)\right)^{i}\right]\left(\mathbf{1}_{\Omega^{2}}-v\right)\right),
$$

where

$$
\Delta_{3 i}^{-1 / 2}=\Delta_{2 i}^{-1 / 2}-v_{i}
$$


Notice that now (again) the sum in $k$ is finite in each cohomological degree. Therefore we can specialize $v$. We will take $v$ to be the vector $\mathbf{1}_{\Omega^{2}}-\mathbf{1}_{\Omega^{1}}$, which is holomorphic by Lemma 3.4.2, and thus $v_{i}=\Delta_{2 i}^{-1 / 2}-\Delta_{1 i}^{-1 / 2}$. In this case, the $\Delta_{3 i}$ specialize to $\Delta_{1 i}$. We have therefore arrived exactly at the reconstruction formula for $\Omega^{1}$. In particular, with the specialization of $v, \Omega^{4}$ is the same as $\Omega^{1}$ and therefore $\Omega^{1}$ is also holomorphic along the discriminant. Hence $\Omega^{1}$ is a suitable local extension of the Frobenius manifold we started with to a convergent CohFT. We note that the extension in not unique but depends on the choice of integration constants in Lemma 3.4.3.

\subsection{Equivalence of relations}

We will prove Theorem 3.3.6 in this section.

First notice that the dimension of a convergent $\operatorname{CohFT} \Omega$ can be increased by one without changing the set of relations by the construction of Example 3.1.7 So we can assume that the CohFTs we are trying to compare have the same dimension.

Next, recall from Section 3.3 that the tautological relations of a semisimple convergent CohFT $\Omega$ are defined via coefficients of the part of the GiventalTeleman classification singular in the discriminant. Therefore the relations do not change when removing the codimension two set of singular points of the discriminant from the Frobenius manifold underlying $\Omega$.

In order to prove Theorem 3.3.6, it is therefore enough to show that the relations coincide for two semisimple, equal dimensional convergent CohFTs $\Omega^{1}, \Omega^{2}$ such that each Frobenius manifold contains a smooth point of the discriminant and is small enough for Theorem 3.4.1 to apply directly to $\Omega^{1}$.

By the proof of Theorem 3.4.1, an extension of the Frobenius manifold underlying $\Omega^{1}$ to a CohFT can be constructed from $\Omega^{2}$ by a holomorphic $R$-matrix and a holomorphic shift. To prove Theorem 3.3.6, it therefore suffices to show that these two operations preserve tautological relations and that the integration constants in the proof of Lemma 3.4.3 can be chosen such that the constructed CohFT coincides with $\Omega^{1}$. We now prove these statements.

Lemma 3.5.1. The R-matrix action by a holomorphic R-matrix preserves tautological relations.

Proof. Let $\Omega^{\prime}$ be obtained from $\Omega$ from the $R$-matrix action of $R$. Then in the description of the $R$-matrix action in Section 3.2, all arguments are holomorphic vector fields on the Frobenius manifold with values in power series in $\psi$-classes. $\Omega_{g, n}^{\prime}$ in each cohomological degree is obtained by a finite sum of push-forwards under the gluing map of products of $\Omega$ (with possibly additional markings) multiplied by monomials in $\psi$ classes and with holomorphic vector fields as arguments. Therefore any singularities of the reconstruction of $\Omega^{\prime}$ are the result of singularities in the reconstruction of $\Omega$. So we can write the relations $q\left(\widetilde{\Omega^{\prime}}{ }_{g, n}\right)$ in terms of the relations from $\Omega$ as of Definition 3.3.1. Since the ideal of relations of $\Omega^{\prime}$ is stable under the tautological maps, we can also express any tautological relation of $\Omega^{\prime}$ as in Definition 3.3.1 in terms of relations from $\Omega$. 
Since $R$-matrices are power series starting with the identity matrix, by using $R^{-1}$, we can also write the relations of $\Omega$ in terms of relations from $\Omega^{\prime}$.

The shift-construction (14) clearly expresses any relation from $\Omega_{g, n}^{4}$ in terms of relations from $\Omega_{g, n+m}^{3}$ for various $m \geq 0$.

Now, we finally show that taking the $R$-matrix of $\Omega^{1}$ is a suitable choice for $R_{1}$ in Lemma 3.4.3. We will argue that otherwise $\Omega^{1}$ or $\Omega^{2}$ would not be defined at the discriminant.

For simplicity, we will make use of the following stability result. It should also be possible to use estimates or congruence properties of intersection numbers instead.

Theorem 3.5.2 (Boldsen [1], Looijenga [17]). Let $M_{g, n} \subset \bar{M}_{g, n}$ be the moduli space of smooth curves. Then, for any $k<\frac{g}{3}$ the vector space $H^{2 k}\left(M_{g, n}\right)$ is freely generated by the set of monomials of cohomological degree $2 k$ in the classes $\kappa_{1}, \ldots, \kappa_{k}, \psi_{1}, \ldots, \psi_{n}$.

We use the local coordinates $t, t_{0}, u_{\geq 3}$ from the proof of Lemma 3.4.3. Let $i$ be the lowest degree in $z$ where $R_{1} R_{2}^{-1}$ is not holomorphic. By the proof of Lemma 3.4.3, the non-holomorphic part in this degree is a constant multiple of the block-diagonal matrix with upper-left block

$$
\left(\begin{array}{cc}
0 & t^{1 / 2} \\
t^{-1 / 2} & 0
\end{array}\right)
$$

and zeros everywhere else. By Theorem 3.5.2 we can consider the $\psi_{1}^{i}$-coefficient of

$$
\left.\Omega_{g, 1}^{1}\left(\frac{\partial}{\partial t_{0}}\right)\right|_{M_{g, 1}}-\left.\Omega_{g, 1}^{2}\left(\frac{\partial}{\partial t_{0}}\right)\right|_{M_{g, 1}}
$$

for large $g$. Its lowest order term when expanded in $t$ is up to a nonzero factor given by

$$
\begin{aligned}
t^{-\frac{1}{2}} \sqrt{t}\left({\sqrt{2 \sqrt{t}^{2 g-2+1-1}}}^{\left.2+{\sqrt{-2 \sqrt{t}^{2 g-2+1-1}}}^{2}\right)}\right. \\
=2^{g-1}\left((\sqrt{t})^{g-1}-(-\sqrt{t})^{g-1}\right),
\end{aligned}
$$

and therefore not holomorphic in $t$ for even $g$. This is a contradiction to the assumption that both $\Omega^{1}$ and $\Omega^{2}$ are defined on the discriminant locus.

\subsection{Global extension}

The local extension Theorem 3.4.1 leaves open the question under what conditions a semisimple Frobenius manifold can (globally) be extended to a CohFT. In Section 3.7.2 we will see that the restrictions put on integration constants of the $R$-matrices in Lemma 3.4 .3 do not always fit together globally. 
Conjecture 3.6.1. Let $M$ be an $N$-dimensional semisimple Frobenius manifold such that it possesses a holomorphic genus one potential $\mathrm{d} G$. Then there exists a convergent CohFT with underlying Frobenius manifold $M$.

On the other hand, when the Frobenius manifold is conformal, such an extension to a CohFT exists by the following simple argument. There is a unique homogeneous solution to the flatness equation (4) and by construction it is meromorphic along the discriminant. Since by Lemma 3.4 .3 all possible solutions are either holomorphic in the discriminant or are multivalued, the homogeneous solution has in fact to be holomorphic.

\subsection{Examples}

\subsubsection{Extending the comparison to non-smooth points on the dis- criminant}

We want to illustrate how the comparison between relations in the proof of Theorem 3.3.6 via an identification of coordinates and vector fields, an $R$-matrix action and a shift, does not directly extend to give a way to explicitly write the relations near a singular point of the discriminant in terms of the $A_{2^{-}}$(3-spin) relations.

Let us consider the comparison between the $A_{2} \times A_{1}$ and $A_{3}$ singularities. We will see that already the identification between points and vector fields behaves badly. This is the simplest example we can consider since in two dimensions the discriminant locus is a union of parallel lines and in particular is non-singular.

The Frobenius manifold of the $A_{3}$-singularity $x^{4} / 4=0$ is based on the versal deformation space

$$
f(x)=\frac{x^{4}}{4}+t_{2} x^{2}+t_{1} x+t_{0} .
$$

Here $t_{0}, t_{1}$ and $t_{2}$ are coordinates on the Frobenius manifold. The ring structure is given by the Milnor ring

$$
\mathbb{C}\left[t_{0}, t_{1}, t_{2}\right][x] / f^{\prime}(x),
$$

where $x=\frac{\partial}{\partial t_{1}}$. The discriminant of the minimal polynomial $f^{\prime}$ of $x$ is $-32 t_{2}^{3}-$ $27 t_{1}^{2}$ and therefore the discriminant locus has a cusp at $t_{1}=t_{2}=0$. The metric is in the basis $\left\{1, x, x^{2}\right\}$ given by

$$
\left(\begin{array}{ccc}
0 & 0 & 1 \\
0 & 1 & 0 \\
1 & 0 & -2 t_{2}
\end{array}\right) .
$$

Therefore the basis $\left\{1, x, x^{2}\right\}$ is flat up to a determinant one basis change.

We go to a sixfold ramified cover of the Frobenius manifold on which we can define the critical points $\zeta_{1}, \zeta_{2}, \zeta_{3}$ of $f(x)$ as holomorphic functions. Let $u_{1}, u_{2}, u_{3}$ be the corresponding critical values. Part of the discriminant locus is 
described by the equation $\zeta_{1}=\zeta_{2}$. Locally we use $\phi:=\zeta_{1}-\zeta_{2}, \zeta_{3}$ and $t_{0}$ as new coordinates. Reexpressing in terms of these coordinates gives

$$
\begin{aligned}
\zeta_{1} & =-\frac{1}{2} \zeta_{3}+\frac{1}{2} \phi, \\
\zeta_{2} & =-\frac{1}{2} \zeta_{3}-\frac{1}{2} \phi, \\
u_{1} & =t_{0}+\frac{3}{64} \zeta_{3}^{4}-\frac{5}{32} \zeta_{3}^{2} \phi^{2}+\frac{1}{8} \zeta_{3} \phi^{3}-\frac{1}{64} \phi^{4}, \\
u_{2} & =t_{0}+\frac{3}{64} \zeta_{3}^{4}-\frac{5}{32} \zeta_{3}^{2} \phi^{2}-\frac{1}{8} \zeta_{3} \phi^{3}-\frac{1}{64} \phi^{4}, \\
u_{1}-u_{2} & =\frac{1}{4} \zeta_{3} \phi^{3} \\
u_{3} & =t_{0}-\frac{3}{8} \zeta_{3}^{4}+\frac{1}{8} \zeta_{3}^{2} \phi^{2} .
\end{aligned}
$$

The idempotents are given by

$$
\begin{aligned}
& \frac{\partial}{\partial u_{1}}=\frac{\left(x-\zeta_{2}\right)\left(x-\zeta_{3}\right)}{\left(\zeta_{1}-\zeta_{2}\right)\left(\zeta_{1}-\zeta_{3}\right)}=\frac{x^{2}+\left(-\frac{1}{2} \zeta_{3}+\frac{1}{2} \phi\right) x-\frac{1}{2} \zeta_{3}^{2}-\frac{1}{2} \zeta_{3} \phi}{-\frac{3}{2} \zeta_{3} \phi+\frac{1}{2} \phi^{2}} \\
& \frac{\partial}{\partial u_{2}}=\frac{\left(x-\zeta_{1}\right)\left(x-\zeta_{3}\right)}{\left(\zeta_{2}-\zeta_{1}\right)\left(\zeta_{2}-\zeta_{3}\right)}=\frac{x^{2}+\left(-\frac{1}{2} \zeta_{3}-\frac{1}{2} \phi\right) x-\frac{1}{2} \zeta_{3}^{2}+\frac{1}{2} \zeta_{3} \phi}{\frac{3}{2} \zeta_{3} \phi+\frac{1}{2} \phi^{2}} \\
& \frac{\partial}{\partial u_{3}}=\frac{\left(x-\zeta_{1}\right)\left(x-\zeta_{2}\right)}{\left(\zeta_{3}-\zeta_{1}\right)\left(\zeta_{3}-\zeta_{2}\right)}=\frac{x^{2}+\zeta_{3} x+\frac{1}{4} \zeta_{3}^{2}-\frac{1}{4} \phi^{2}}{\frac{9}{4} \zeta_{3}^{2}-\frac{1}{4} \phi^{2}}
\end{aligned}
$$

so that they become after normalization

$$
\begin{gathered}
\frac{x^{2}+\left(-\frac{1}{2} \zeta_{3}+\frac{1}{2} \phi\right) x-\frac{1}{2} \zeta_{3}^{2}-\frac{1}{2} \zeta_{3} \phi}{\sqrt{-\frac{3}{2} \zeta_{3} \phi+\frac{1}{2} \phi^{2}}}, \frac{x^{2}+\left(-\frac{1}{2} \zeta_{3}-\frac{1}{2} \phi\right) x-\frac{1}{2} \zeta_{3}^{2}+\frac{1}{2} \zeta_{3} \phi}{\sqrt{\frac{3}{2} \zeta_{3} \phi+\frac{1}{2} \phi^{2}}}, \\
\frac{x^{2}+\zeta_{3} x+\frac{1}{4} \zeta_{3}^{2}-\frac{1}{4} \phi^{2}}{\sqrt{\frac{9}{4} \zeta_{3}^{2}-\frac{1}{4} \phi^{2}}} .
\end{gathered}
$$

For $A_{2} \times A_{1}$, let us assume that $u_{3}$ corresponds to the $A_{1}$-direction and that the norm of the idempotent in that direction is one. We can write

$$
u_{1}=x_{0}-\frac{2}{3}\left(-x_{1}\right)^{3 / 2}, \quad u_{2}=x_{0}+\frac{2}{3}\left(-x_{1}\right)^{3 / 2},
$$

where $x_{0}$ and $x_{1}$ are flat coordinates corresponding to $t_{0}$ and $t_{1}$ in Example 2.2.1.

We should therefore identify

$$
\phi \stackrel{!}{=}-2\left(\frac{2}{3}\right)^{1 / 3} \zeta_{3}^{-1 / 3} \sqrt{-x_{1}} .
$$

Let us consider how we identify the $A_{3}$-singularity basis $\left\{1, x, x^{2}\right\}$ and the flat basis of $A_{2} \times A_{1}$ via the identification of their normalized idempotents. If 
we write the identity of $A_{2} \times A_{1}$ in terms of $\left\{1, x, x^{2}\right\}$, the $x^{2}$-coefficient is

$$
\begin{gathered}
\frac{\sqrt{2 \sqrt{-x_{1}}}}{\sqrt{-\frac{3}{2} \zeta_{3} \phi+\frac{1}{2} \phi^{2}}}+\frac{\sqrt{-2 \sqrt{-x_{1}}}}{\sqrt{\frac{3}{2} \zeta_{3} \phi+\frac{1}{2} \phi^{2}}}+\frac{1}{\sqrt{\frac{9}{4} \zeta_{3}^{2}-\frac{1}{4} \phi^{2}}} \\
=\frac{2 \zeta_{3}^{1 / 6}}{\sqrt{-3 c \zeta_{3}+c \phi}+\frac{2 \zeta_{3}^{1 / 6}}{\sqrt{3 c \zeta_{3}+c \phi}}+\frac{2}{\sqrt{9 \zeta_{3}^{2}-\phi^{2}}},}
\end{gathered}
$$

where

$$
c=-2\left(\frac{2}{3}\right)^{1 / 3}
$$

The coefficient is well-defined on generic points of the part $\zeta_{1}=\zeta_{2}(\phi=0)$ of the discriminant, but when fixing some $\phi \neq 0$ the function has a singularity at $\zeta_{3}=0$.

\subsubsection{Obstructions to extending $R$-matrices}

We want consider the class of two-dimensional Frobenius manifolds with flat coordinates $t_{0}, t$, flat metric

$$
\eta=\left(\begin{array}{ll}
0 & 1 \\
1 & 0
\end{array}\right)
$$

and quantum product

$$
\left(\frac{\partial}{\partial t}\right)^{2}=f \frac{\partial}{\partial t_{0}}
$$

for a holomorphic function $f(t)$. The corresponding Gromov-Witten potential is

$$
\frac{1}{2} t_{0}^{2} t+F
$$

where $F(t)$ is a third anti-derivative of $f(t)$.

The differential equation satisfied by the $R$-matrix in flat coordinates can be made explicit:

$$
\left[R,\left(\begin{array}{ll}
0 & f \\
1 & 0
\end{array}\right)\right]+z \dot{R}+z \frac{\dot{f}}{4 f}\left(\begin{array}{cc}
-1 & 0 \\
0 & 1
\end{array}\right) R=0
$$

We first want to show that for any solution $R$, the $z^{1}$-coefficient is not holomorphic for all $f$. For this we set

$$
R=\left(\begin{array}{ll}
1+a z & 0+b z \\
0+c z & 1+d z
\end{array}\right)+O\left(z^{2}\right)
$$

From (15) in degree $z^{1}$ we obtain

$$
b-f c-\frac{\dot{f}}{4 f}=0, \quad a=d .
$$


From (15) in degree $z^{2}$, we see that $a=d$ is an integration constant. We obtain an interesting differential equation for $c$ :

$$
2 f \dot{c}+\dot{f} c+\frac{\ddot{f}}{4 f}-\frac{5 \dot{f}^{2}}{16 f^{2}}=0
$$

If we substitute

$$
c=\frac{\gamma}{f}-\frac{5}{48} \frac{\dot{f}}{f^{2}}
$$

it becomes

$$
2 \dot{\gamma}-\frac{\dot{f}}{f} \gamma+\frac{\ddot{f}}{24 f}=0 .
$$

So $\gamma$ is determined up to a multiple of a root of $f$ and in particular, if $f$ has somewhere a simple zero, there exists at most one solution meromorphic on all of $\mathbb{C}^{2}$.

If $f$ is linear, $\gamma=0$ is clearly a holomorphic solution. If $f$ is quadratic with non-vanishing discriminant, there is still a holomorphic solution. For example for

$$
f(t)=t(t+1)
$$

the solution is

$$
\gamma=\frac{t}{6}+\frac{1}{12}
$$

In larger degree, we stop having meromorphic solutions. In the example

$$
f(t)=t\left(t^{2}-1\right)
$$

after substituting

$$
\gamma=f \delta+\frac{t}{8}
$$

we arrive at the differential equation

$$
\left(t^{2}-1\right) 2 t \dot{\delta}+\left(3 t^{2}-1\right) \delta+\frac{1}{8}=0 .
$$

We see that $\delta$ is meromorphic in $t$ if and only it is so in $u:=t^{2}$. In the new variable the differential equation is

$$
4 u(u-1) \delta^{\prime}+(3 u-1) \delta+\frac{1}{8}=0 .
$$

From generic semisimplicity we also know that $\delta$ has to be holomorphic except for $u=0$ and $u=1$. Around $u=0$ and $u=1$ there are unique meromorphic solutions

$$
\frac{1}{8} \sum_{i=0}^{\infty} \frac{4 i+3}{4 i+1} u^{i}, \quad-\frac{1}{16} \sum_{i=0}^{\infty} \frac{4 i+3}{4 i+2}(1-u)^{i},
$$

but these obviously do not agree (consider $u=\frac{1}{2}$ ). 
We now want to check that the corresponding genus one potential will also be singular, in agreement with Conjecture 3.6.1 For this, we look at the case when $a=d=0$, and compute the codimension one part of the reconstructed CohFT on $\bar{M}_{1,1}$ with an $\frac{\partial}{\partial t}$-insertion. From the trivial graph, we obtain the contribution

$$
-2\left(\gamma+\frac{7}{48} \frac{\dot{f}}{f}\right) \psi_{1}+2\left(\gamma-\frac{5}{48} \frac{\dot{f}}{f}\right) \kappa_{1}
$$

and in addition we have the contribution

$$
2 \gamma+\frac{2}{48} \frac{\dot{f}}{f}
$$

of the irreducible divisor $\delta_{0}$. From

$$
\int_{\bar{M}_{1,1}} \psi_{1}=\int_{\bar{M}_{1,1}} \kappa_{1}=\frac{1}{12} \int_{\bar{M}_{1,1}} \delta_{0}=\frac{1}{24}
$$

we see that the correlator equals $\gamma$, which is not holomorphic on all of the Frobenius manifold.

\subsection{Other relations from cohomological field theories}

For a convergent CohFT depending on additional parameters, there are ways different from Definition 3.3.1 to obtain tautological relations from the reconstruction of semisimple CohFTs. We will consider here the example of the equivariant Gromov-Witten theory of a toric variety, which is dependent on equivariant and Novikov parameters. We will see that the additional relations are still a consequence of Pixton's relations.

Let $T=\left(\mathbb{C}^{*}\right)^{m}$ and let $H_{T}^{*}(\mathrm{pt})=H^{*}(B T)=\mathbb{C}\left[\lambda_{1}, \ldots, \lambda_{m}\right]$ be the $T$ equivariant cohomology ring of a point. Let $X$ be an $m$-dimensional smooth, toric variety with a basis $\left\{p_{1}, \ldots, p_{N}\right\}$ of its cohomology, which we can also lift to a basis in $T$-equivariant cohomology. Let $\beta_{1}, \ldots, \beta_{N}$ be the dual homology basis. The Novikov ring is a completion of the semigroup ring of effective classes $\beta \in$ $H_{2}(X ; \mathbb{Z})$. We use $q^{\beta}$ to denote the generator corresponding to a $\beta \in H_{2}(X ; \mathbb{Z})$.

A family of $N$-dimensional CohFTs on the state space $H_{\mathbb{C}^{*}}^{*}(X)$ can be defined by setting

$$
\Omega_{g, n}\left(\alpha_{1}, \ldots, \alpha_{n}\right)=\sum_{\beta} q^{\beta} p_{*}\left(\prod_{i=1}^{n} \mathrm{ev}_{i}^{*}\left(\alpha_{i}\right) \cap\left[\bar{M}_{g, n}(X ; \beta)\right]^{v i r}\right),
$$

where the sum ranges over all effective classes $\beta \in H_{2}(X ; \mathbb{Z}), p$ is the projection from the moduli space of stable maps to $\bar{M}_{g, n}$, and $\mathrm{ev}_{i}$ is the $i$ th evaluation map. At this point $\Omega_{g, n}$ is just a power series in the Novikov variables.

Using virtual localization with respect to the $T$-action on $X$, the CohFT $\Omega_{g, n}$ can be effectively computed. The corresponding $R$-matrix is however only defined after localizing the equivariant parameters $\lambda_{1}, \ldots, \lambda_{m}$. This gives rise 
to tautological relations defined similarly to those of Definition 3.3.1. The reconstruction formula (which in this case is a consequence of virtual localization) gives an expression $\widetilde{\Omega}_{g, n}^{\text {loc }}$ for $\Omega_{g, n}$ which does not seem to admit the nonequivariant limit $\lambda_{i} \rightarrow 0$. On the other hand, the well-defined non-equivariant Gromov-Witten theory of $X$ must be recovered in this limit. The necessary cancellation of poles in the equivariant parameters gives rise to tautological relations. In the example of $X=\mathbb{P}^{1}$, the resultant relations actually imply Pixton's relations [15, 13.

We now compare these relations from equivariant localization to the relations considered in Definition 3.3.1. From results [12] of Iritani, it follows that the sum over $\beta$ defining $\Omega_{g, n}$ converges in a neighborhood of the origin, and that the CohFT induces a convergent CohFT varying analytically on the additional Novikov and equivariant parameters. We can therefore view $\Omega_{g, n}$ as valued on the space of functions $\mathcal{O}_{U}$ on an open neighborhood $U$ of the orgin in the product of the Frobenius manifold and the space of possible Novikov and equivariant parameters. By mirror symmetry (see [12, Section 6]), it follows also that the coefficients of the $R$-matrix are valued in $\mathcal{O}_{U \backslash D}$ where $D \subset U$ is a discriminant locus. Therefore the reconstruction gives elements $\widetilde{\Omega}_{g, n} \in \mathcal{S}_{g, n} \otimes\left(V^{*}\right)^{\otimes n} \otimes \mathcal{O}_{U \backslash D}$. As in Definition 3.3.1, projecting to $\mathcal{O}_{U \backslash D} / \mathcal{O}_{U}$ yields tautological relations. Since for any value of the Novikov and equivariant parameters, the CohFT is generically semisimple, we can apply Theorem 3.3.6 to see that all these tautological relations are consequences of Pixton's relations. Restricting $\widetilde{\Omega}_{g, n}$ to the origin on the Frobenius manifold and Taylor expanding in the Novikov

parameters, we recover $\widetilde{\Omega}_{g, n}^{\text {loc }}$. It follows that the tautological relations from considering the non-equivariant limit are also consequences of Pixton's relations.

Remark 3.8.1. A similar strategy should also work for toric orbifolds. The special case of $\mathbb{P}^{1}$ with two orbifold points is used in [2].

\section{References}

[1] Søren K. Boldsen. Improved homological stability for the mapping class group with integral or twisted coefficients. Math. Z., 270(1-2):297-329, 2012. arXiv:0904.3269.

[2] Emily Clader and Felix Janda. Pixton's double ramification cycle relations, 2016. arXiv:1601.02871.

[3] Tom Coates and Hiroshi Iritani. On the convergence of Gromov-Witten potentials and Givental's formula. Michigan Math. J., 64(3):587-631, 2015. arXiv:1203.4193.

[4] Boris Dubrovin. Geometry of 2D topological field theories. In Integrable systems and quantum groups (Montecatini Terme, 1993), volume 1620 of Lecture Notes in Math., pages 120-348. Springer, Berlin, 1996. arXiv:hep-th/9407018 
[5] Carel Faber and Rahul Pandharipande. Relative maps and tautological classes. J. Eur. Math. Soc. (JEMS), 7(1):13-49, 2005. arXiv:math/0304485.

[6] Alexander Givental. Elliptic Gromov-Witten invariants and the generalized mirror conjecture. In Integrable systems and algebraic geometry (Kobe/Kyoto, 1997), pages 107-155. World Sci. Publ., River Edge, NJ, 1998. arXiv:math/9803053.

[7] Alexander B. Givental. Gromov-Witten invariants and quantization of quadratic Hamiltonians. Mosc. Math. J., 1(4):551-568, 645, 2001. Dedicated to the memory of I. G. Petrovskii on the occasion of his 100th anniversary.

[8] Alexander B. Givental. Semisimple Frobenius structures at higher genus. Internat. Math. Res. Notices, (23):1265-1286, 2001. arXiv:math/0008067.

[9] Tom Graber and Rahul Pandharipande. Constructions of nontautological classes on moduli spaces of curves. Michigan Math. J., 51(1):93-109, 2003. arXiv:math/0104057.

[10] Claus Hertling. Multiplication of the tangent bundle. arXiv:math.AG/9910116.

[11] Claus Hertling. Frobenius manifolds and variance of the spectral numbers. In New developments in singularity theory (Cambridge, 2000), volume 21 of NATO Sci. Ser. II Math. Phys. Chem., pages 235-255. Kluwer Acad. Publ., Dordrecht, 2001. arXiv:math/0007187.

[12] Hiroshi Iritani. Convergence of quantum cohomology by quantum Lefschetz. J. Reine Angew. Math., 610:29-69, 2007. arXiv:math/0506236.

[13] Felix Janda. Tautological relations in moduli spaces of weighted pointed curves, 2013. arXiv:1306.6580

[14] Felix Janda. Comparing tautological relations from the equivariant Gromov-Witten theory of projective spaces and spin structures, 2014. arXiv:1407.4778,

[15] Felix Janda. Relations on $\bar{M}_{g, n}$ via equivariant Gromov-Witten theory of $\mathbb{P}^{1}$. Algebr. Geom., 4(3):311-336, 2017. arXiv:1509.08421.

[16] Maxim Kontsevich and Yuri Manin. Gromov-Witten classes, quantum cohomology, and enumerative geometry. In Mirror symmetry, II, volume 1 of AMS/IP Stud. Adv. Math., pages 607-653. Amer. Math. Soc., Providence, RI, 1997. arXiv:hep-th/9402147.

[17] Eduard Looijenga. Stable cohomology of the mapping class group with symplectic coefficients and of the universal Abel-Jacobi map. J. Algebraic Geom., 5(1):135-150, 1996. arXiv:alg-geom/9401005. 
[18] Todor Milanov. The Eynard-Orantin recursion for simple singularities. Commun. Number Theory Phys., 9(4):707-739, 2015. arXiv:1303.3103.

[19] David Mumford. Towards an enumerative geometry of the moduli space of curves. In Arithmetic and geometry, Vol. II, volume 36 of Progr. Math., pages 271-328. Birkhäuser Boston, Boston, MA, 1983.

[20] Rahul Pandharipande, Aaron Pixton, and Dimitri Zvonkine. Relations on $\bar{M}_{g, n}$ via 3-spin structures. J. Amer. Math. Soc., 28(1):279-309, 2015. arXiv:1303.1043.

[21] Rahul Pandharipande, Aaron Pixton, and Dimitri Zvonkine. Tautological relations via $r$-spin structures, 2016. arXiv:1607.00978.

[22] Dan Petersen and Orsola Tommasi. The Gorenstein conjecture fails for the tautological ring of $\overline{\mathcal{M}}_{2, n}$. Invent. Math., 196(1):139-161, 2014. arXiv:1210.5761

[23] Aaron Pixton. Conjectural relations in the tautological ring of $\bar{M}_{g, n}, 2012$. arXiv:1207.1918

[24] Constantin Teleman. The structure of 2D semi-simple field theories. Invent. Math., 188(3):525-588, 2012. arXiv:0712.0160.

Department of Mathematics

University of Michigan

2074 East Hall

530 Church Street

Ann Arbor

MI-48109

USA 